\title{
Técnicas activas para la enseñanza de la educación superior en el proceso pedagógico intra-aula
}

\section{Active techniques for teaching higher education in the intra-classroom pedagogical process}

\section{Técnicas activas en la educación superior.}

Fabrina Monserrate Cedeño Mendoza, MSc. ${ }^{(1)}$

Gilberto Antonio Jarre Cedeño, MSc. ${ }^{(2)}$

Raquel Inés Macías Cedeño, MSc. ${ }^{(3)}$

Gilberto Rafael Jarre Vera MSc. ${ }^{(4)}$

(1) Universidad Técnica de Manabí, Instituto de Ciencias Básicas, Portoviejo - Manabí - Ecuador fmcedeno@utm.edu.ec ORCID: https://orcid.org/0000-0002-6829-6683

(2) Universidad Técnica de Manabí, Facultad de Ingeniería Agrícola, Portoviejo - Manabí - Ecuador giarre@utm.edu.ec ORCID: https://orcid.org/0000-0003-1087-5938

(3) Unidad Educativa Sucre, 24 de Mayo, Manabí, Ecuador, raquelmacias1998@gmail.com ORCID: https://orcid.org/0000-0002-7644-5829

(4) Universidad Técnica de Manabí, Facultad de Matemáticas, Físicas y Químicas, Portoviejo - Manabí - Ecuador grjarre@utm.edu.ec ORCID: https://orcid.org/0000-0001-9915-0823

Contacto: fmcedeno@utm.edu.ec

Recibido: 18-02-2020

Aprobado: 01-04-2020

\section{Resumen}

El presente es un estudio que enfatiza la investigación de técnicas para la enseñanza de la educación superior en el proceso pedagógico intra-aula, el mismo que se respalda bajo los objetivos: a) Incidencia y monitoreo de la aplicación de técnicas activas en el proceso pedagógico de la educación superior, b) Evaluación de técnicas activas en el proceso pedagógico intra-aula. Básicamente hacer que el estudiante de educación superior piense productivamente, desarrollando su razonamiento, enseñándole a enfrentar situaciones nuevas, brindando la oportunidad de involucrarse en la clase para que el aprendizaje sea más interesante, y de esta manera equipar al estudiante con estrategias para resolver problemas, proporcionándole técnicas que sirvan de ayuda en su perfeccionamiento profesional. El tratamiento metodológico de la investigación está basado en las siguientes modalidades: bibliográfica, de Campo, y Cuasi- experimental. Además, se usó los siguientes tipos de investigación: exploratorio, el descriptivo, el analítico, el sintético. Los resultados exteriorizan que los docentes de educación superior si aplican técnicas pedagógicas activas en los diferentes procesos de la gestión educativa, llegando a la conclusión que los docentes si aplican técnicas en el proceso pedagógico intra-aula, las mismas que facilitan el aprendizaje significativo de los estudiantes garantizando la calidad de educación, esto fue también exteriorizado por los estudiantes quienes afirman que la aplicación de técnicas por parte de los docentes ayuda a resolver problemas de la vida cotidiana.

Palabras Claves: Técnicas activas, proceso pedagógico, educación superior.

\section{Sumary}

This is a study that emphasizes the investigation of techniques for teaching higher education in the intraclassroom pedagogical process, which is supported by the objectives: a) Incidence and monitoring of the application of active techniques in the pedagogical process of higher education, b) Evaluation of active techniques in the intra-classroom pedagogical process. Basically make the higher education student think 
productively, developing his reasoning, teaching him to face new situations, providing the opportunity to get involved in the class to make learning more interesting, and thus equip the student with problem solving strategies, providing you with techniques that help in your professional development. The methodological treatment of the research is based on the following modalities: bibliographic, Field, and Quasiexperimental. In addition, the following types of research were used: exploratory, descriptive, analytical, synthetic. The results show that higher education teachers do apply active pedagogical techniques in the different educational management processes, concluding that teachers do apply techniques in the intra-classroom pedagogical process, which facilitate the meaningful learning of students guaranteeing the quality of education, this was also externalized by the students who affirm that the application of techniques by teachers helps solve problems of daily life.

Keywords: Active techniques, pedagogical process, higher education.

\section{Introducción}

Debido a las insuficiencias didácticas en la enseñanza de la educación superior, se torna interesante efectuar una reflexión referente lo que sucede con la población estudiantil superior sobre la enseñanza que tiene por finalidad incorporar valores y desarrollar actitudes, de manera que obtenga un concepto claro y amplio y para ello se requiere el uso de técnicas activas que permitan desarrollar las capacidades para percibir, comprender, asociar, analizar e interpretar los conocimientos adquiridos para enfrentar su entorno (Chevallard, Bosch, \& Gascón, 1996). El docente debe proporcionar al estudiante una orientación general sobre las asignaturas, con el objeto de facilitar y orientar el estudio donde versará su vida cotidiana, debe proveer de los métodos de razonamiento básico, requerido así mismo, para plantear algunos ejercicios a resolver cuya ejecución le permitirá afianzar sus conocimientos (Artigue, Douady, Moreno, \& Gómez, 1995).

La inteligencia no es algo fijo sino modificable, el enfoque actual de la inteligencia no la identifica tanto con los conocimientos sino con el repertorio de habilidades que permiten actuar inteligentemente. La inteligencia no es tanto una entidad cuanto un conjunto de habilidades; es más bien un sistema abierto y como tal puede mejorar (Edel Navarro, 2003).

La nueva concepción de las técnicas de aprendizaje concibe al estudiante como un ser activo que construye sus propios conocimientos inteligentemente, es decir, utilizando las estrategias que posee. Mientras que en la concepción anterior el profesor se limitaba a transmitir contenidos, ahora su cometido principal es ayudar a aprender. Y como aprender es construir conocimientos, es decir, manejar, organizar, estructurar y comprender la información, o lo que es lo mismo, poner en contacto las habilidades del pensamiento con los datos informativos, aprender es aplicar cada vez mejor las habilidades intelectuales a los contenidos del aprendizaje (Tierno, 2012). Aprender es pensar; y enseñar es ayudar al alumno a pensar, mejorando diariamente las estrategias o habilidades de ese pensamiento.

Las técnicas activas de aprendizaje conducen al estudiante hacia la memoria comprensiva y lo convierten en el principal protagonista del proceso educativo. Estas técnicas que se las realizan con la ayuda del computador, se fundamentan en el razonamiento permanente, para descubrir las relaciones causa-efecto de las cosas y arribar hacia un aprendizaje que les sirva para la vida y, se basan en el desarrollo del pensamiento crítico y del pensamiento creativo, la actividad del aprendizaje está centrada en la actividad del estudiante (Beltrán, 1996).

Con la aplicación de estas técnicas se pretende desarrollar la agilidad mental, el análisis y la síntesis, la actitud creativa e investigativa y la participación libre y espontánea, son una herramienta, que para su aplicación se debe considerar aspectos como: característica y contenido de la asignatura, la disposición del grupo, el espacio físico disponible, los recursos didácticos necesarios y la habilidad del profesor para su manejo. (Gómez Gómez, s.f.). Cada técnica tiene sus propias características, pero ello no impide que el docente logre adecuar, modificar o adaptar las técnicas de acuerdo a sus necesidades y circunstancias.

De ahí resultan las técnicas de estudio o estrategias de estudio, son distintas perspectivas aplicadas al aprendizaje, generalmente son críticas para alcanzar el éxito en la educación superior. Hay una variedad de técnicas de estudio, que pueden enfocarse en el proceso de organizar, tomar y retener nueva información, o superar exámenes, estas técnicas incluyen mnemotecnias, que ayudan a la retención de listas de información, y toma de notas efectiva (Fernández De Haro, s.f.).

Es una manera formulada que de este modo y bajo esta denominación, se integran y agrupan técnicas directamente implicadas en el propio proceso del estudio; tales como la planificación de dicha actividad, el subrayado, el resumen, la elaboración de esquemas, el repaso, entre otros; así como otras estrategias que 
tienen un carácter más complementario, como pueden ser la toma de apuntes o la realización de proyectos de problematización.

La psicología del aprendizaje, particularmente la concepción "constructivista" procedente de la psicología cognitiva, como la práctica educativa, coinciden en considerar el modelado y el moldeado docente como las estrategias didácticas más idóneas a la hora de promover un aprendizaje eficaz y profundo de dichas estrategias, aunque frecuentemente se le deja al estudiante y a su red personal de soporte, se está incrementando la enseñanza de las técnicas de estudio a nivel de la escuela secundaria y educación superior (Coll, y otros, 1999). Existe disponible un gran número de libros y sitios web, que abarcan desde trabajos acerca de técnicas específicas, tales como los libros de Tony Buzan acerca de mapas mentales, hasta guías generales para un estudio exitoso.

Más ampliamente, una técnica que mejora la habilidad de una persona para estudiar y superar exámenes puede ser denominada técnica de estudio, y esto puede incluir técnicas de administración del tiempo y motivacionales; las técnicas de estudio son técnicas discretas que pueden ser aprendidas, generalmente en un período corto, y ser aplicadas a todos o casi todos los campos de estudio, en consecuencia debe distinguírseles de las que son específicas para un campo particular de estudio, por ejemplo la música o la tecnología, y de habilidades inherentes al estudiante, tales como aspectos de inteligencia y estilo de aprendizaje (Serrano Gónzález-Tejero \& Pons Parra, 2011).

Es útil mencionar que los tipos de aprendizaje memorístico y significativo son los extremos de un continuo en el que ambos coexisten en mayor o menor grado $\mathrm{y}$ en la realidad no podemos hacerlos excluyentes. Muchas veces aprendemos algo en forma memorista y tiempo después, gracias a una lectura o una explicación, aquello cobra significado para nosotros; o lo contrario, podemos comprender en términos generales el significado de un concepto, pero no somos capaces de recordar su definición o su clasificación de acuerdo a las técnicas aplicadas (Sebastián, Ballesteros, \& Sánchez García, s.f.).

Ahora bien, esto equivale a decir que las técnicas activas son una variable hipotética, un constructo invisible que enlaza las dos variables dependiente e independiente dejando en la oscuridad la naturaleza de los procesos del aprendizaje.

Para algunos autores la presencia de estas dos variables es suficiente para explicar y planificar el aprendizaje.
Para otros, en cambio, es precisamente ese núcleo central invisible del aprendizaje lo que interesa conocer y desvelar para introducir en las técnicas de aprendizaje mejoras cualitativas, en este punto es donde empiezan a separarse los diversos enfoques psicológicos.

Los objetivos planteados para esta investigación se acentuaron en: a) Incidencia y monitoreo de la aplicación de técnicas activas en el proceso pedagógico de la educación superior, b) Evaluación de técnicas activas en el proceso pedagógico intra-aula.

Todo ello, acompañado por una serie de estrategias meta cognitivas, monitorización de la ejecución de la tarea que en ellas están presentes de forma más o menos consciente, en todo este proceso del estudio: auto cuestionamiento, uso de analogías, supervisión y regulación de la propia ejecución; el mismo que sería el propósito primordial en el que el docente al seleccionar la técnica adecuada deberá tener en cuenta en primer lugar estos objetivos que se persiguen, los referentes teóricos-conceptuales de la asignatura y la característica de los estudiantes.

\section{Materiales y métodos}

El trabajo de investigación científica se apoyó en el método bibliográfico o de recopilación teórica porque se indagó como sustentación las informaciones relacionadas para las variables dependientes e independientes en fuentes como el internet, textos, acuerdos internaciones y los proyectos de similar contenido que existen en las bibliotecas de los centro de educación superior, de igual forma se empleó la metodología de campo porque esta se constituye en la aplicación práctica de la teoría para comprobar si ella funciona o no en la propuesta objeto de investigación mediante encuestas, entrevistas y guías de observaciones.

Así mismo la metodología cuasi-experimental porque a pesar de que el tema ya ha sido estudiado en otros escenarios con diferentes realidades; este se realizó en un ambiente distinto como lo es en los estudiantes de la educación superior. Así mismo la población fue de 130 encuestados entre estudiantes y docentes; el instrumento de encuesta está basado en un total de 3 preguntas para docentes y 3 preguntas para estudiantes con 3 ítems como respuesta y, se efectuó 4 entrevistas a autoridades.

Con la aplicación del método exploratorio que se constituye en el nivel más elemental de la investigación, se recopilaron los primeros datos del objeto de la investigación. Se aplicó el método 
descriptivo en la fundamentación teórica de las dos variables que vamos a describir.

Para analizar los datos estadísticos que se obtuvo del trabajo de campo se utilizó el método analítico que permite explicar e interpretar toda la información cuantitativa.

\section{Resultados}

En cuanto a la aplicación de técnicas activas en el proceso pedagógico intra-aula de la educación superior, alcanzando los siguientes resultados: de los docentes encuestados, el $76,67 \%$ escogieron la alternativa a) excelente, el $23,33 \%$ con la alternativa b) muy buena; con respecto a los estudiantes el $35,8 \%$ considera que la aplicación de técnicas activas es excelente, el 55,56\% muy buena, y el $8,64 \%$ buena; los resultados de los docentes evidencian que las técnicas en el proceso pedagógico intra-aula es excelente, en cambio los estudiantes consideran como muy buena la aplicación de técnicas activas por parte de los docentes de educación superior.

Con respecto a la pregunta que hace referencia a la aplicación de técnicas activas y su incidencia en el proceso pedagógico, los docentes manifestaron: el $76,67 \%$ respondieron mucho, y el $23,33 \%$ la opción poco; en cuanto a la encuesta efectuada a los estudiantes el $91,36 \%$ con un sí y con un 8,64\% respondieron no. En correspondencia con los datos obtenidos, se deduce, que los docentes de educación superior si aplican técnicas o estrategias en el proceso pedagógico.

A través de la interrogante se pretendió conocer la evaluación de técnicas activas durante el proceso pedagógico intra-aula, los resultados son los siguientes: de los docentes encuestados el $100 \%$ respondió que si aplica la evaluación de técnicas activas; los estudiantes respondieron con el $74,07 \%$ que sí, con el $23,46 \%$ afirmaron que no y con el 2,47\% escogieron la opción nada. En concordancia con los resultados obtenidos se evidencia que si evalúan las técnicas activas o estrategias en el proceso pedagógico de la educación superior y son aplicadas por los docentes.

La entrevista realizada a autoridades de educación superior se manifiesta en el hacer que el estudiante piense productivamente, desarrollando su razonamiento, enseñándole a enfrentar situaciones nuevas, brindando la oportunidad de involucrarse en la clase para que el aprendizaje sea más interesante, y de esta manera equipar al estudiante con estrategias para resolver problemas, proporcionándole una buena base; es decir que se debe potenciar con herramientas metacognitivas que permita al estudiante aprehender para la vida.

\section{Discusión}

Un aprendizaje de calidad en la educación superior se aprecia cuando los contenidos son utilizados correctamente al momento de solucionar problemas específicos o problemas de la vida cotidiana. Ausubel (1983), "El alumno debe manifestar una disposición para relacionar, lo sustancial y no arbitrariamente el nuevo material con su estructura cognoscitiva, como que el material que aprende es potencialmente significativo para él, es decir, relacionable con su estructura de conocimiento sobre una base no arbitraria" planteando la siguiente interrogante ¿Por qué el uso de mapas conceptuales para las clases? La elaboración de mapas por parte del docente y la utilización de éstos en las aulas permite un aprendizaje de los contenidos de manera constructiva y significativa, así como un adecuado almacenamiento del material en la estructura cognitiva del estudiante para disponer de ellos cuando se requiera.

$\mathrm{Al}$ respecto de la aplicación de las técnicas activas en el proceso pedagógico de la educación superior, la teoría de la asimilación de (Ausubel, 1983) sostiene que "la interacción entre los nuevos conceptos y los ya existentes se realiza siempre de forma transformadora y que el producto final supone una modificación tanto de las nuevas ideas aprendidas, como de los conocimientos ya existentes, el alumno debe manifestar una disposición para relacionar sustancial y no arbitrariamente el nuevo material con su estructura cognoscitiva, como que el material que aprende es potencialmente significativo para él, es decir, relacionable con su estructura de conocimiento sobre una base no arbitraria".

Lo anterior presupone que el material sea potencialmente significativo, esto implica que el material de aprendizaje pueda relacionarse de manera no arbitraria y sustancial, es decir no al pie de la letra; con alguna estructura cognoscitiva específica del estudiante, la misma que debe poseer "significado lógico" es decir, ser relacionable de forma intencional $\mathrm{y}$ sustancial con las ideas correspondientes y pertinentes que se hallan disponibles en la estructura cognitiva del alumno, este significado se refiere a las características inherentes del material que se va aprender y a su naturaleza.

Para lograr aprendizajes significativos se requiere el uso de técnicas pedagógicas activas, seleccionando las más adecuadas a los objetivos que pretendemos y sobre todo deben ser variadas, es decir, comprobando las 
diversas técnicas existentes, en los diferentes procesos de la gestión educativa. Por lo cual Ausubel (1976, p. 57) "Para que ocurra realmente el aprendizaje significativo no basta con que el material nuevo sea intencionado y relacionable sustancialmente con las ideas correspondientes en el sentido abstracto del término. Es necesario también que tal contenido ideativo pertinente exista en la estructura cognoscitiva del alumno en particular"

De tal manera (Ausubel, 1963) "El alumno construye su conocimiento a través de procesos de diferenciación e integración entre la estructura cognitiva y la estructura del medio" por su parte, propone un modelo de enseñanza por exposición, es decir, explicación o presentación de hechos y hace una defensa del aprendizaje por recepción para explicar el progreso en el conocimiento, con la instrucción se crean situaciones óptimas para el aprendizaje.

Desde esta perspectiva, abordamos la enseñanza de los nuevos contenidos como integración en las redes y esquemas conceptuales previos de los alumnos más aun teniendo en cuenta que, en nuestro caso, el estudiante debe contar ya con conocimientos previos, defendemos la figura del docente como facilitador de los elementos necesarios para que se produzca el aprendizaje, pero no de forma exclusiva, ya que esa misma función la puede realizar un estudiante o un grupo de estudiantes; y, por último, valoramos el uso de las estrategias cognitivas, metacognitivas y sociales que desarrollan el conocimiento procesual y su propia autonomía en el aprendizaje.

(Ausubel, Novack. 1978) "las vías más promisorias para mejorar el aprendizaje escolar, consiste en mejorar los materiales de enseñanza" En torno a lo cual, pueden relacionarse dinámicas estrategias, que exalten la capacidad metacognitivas del educando. Con ello, la habilidad para controlar los procesos, organizarlos, revisarlos, modificarlos, en función de las exigencias del contexto. En este sentido, la figura del docente es importante por su rol de mediador en el proceso educativo, aportando diseños creativos y sobre todo técnicas activas que le permitan al estudiante una mejor aplicación en su proceso comunicacional.

Por consiguiente, Ausubel (1983, p. 55) "Cuando el significado potencial se convierte en contenido cognoscitivo nuevo, diferenciado e idiosincrático dentro de un individuo en particular como resultado del aprendizaje significativo, se puede decir que ha adquirido un "significado psicológico" de esta forma el emerger del significado psicológico no solo depende de la representación que el alumno haga del material lógicamente significativo, " sino también que tal alumno posea realmente los antecedentes ideativo necesarios" en su estructura cognitiva". Siendo las estrategias un factor que contribuyen a que ocurra el aprendizaje, y en la que se fundamenta la labor educativa, en este sentido, el docente deberá desempeñar su labor en principios de aprendizaje bien establecido, con que podrá racionalmente elegir nuevas técnicas activas y estrategias de enseñanza en el proceso pedagógico intra-aula y así mejorar la efectividad de su labor.

El aprendizaje significativo, de acuerdo con (Ausubel, 1983), forma en el educando herramientas metacognitivas que permiten determinar la organización de su estructura cognitiva-conductual previa, y así orientarlo en la formación de saberes teórico, prácticos y formativos en su trayecto por la educación superior.

\section{Conclusiones}

De acuerdo con los resultados estadísticos de la encuesta con respecto a investigar la aplicación de técnicas activas para la enseñanza de la educación superior en el proceso pedagógico intra-aula, se pudo concluir que los docentes si aplican técnicas en el proceso pedagógico intra-aula, las mismas que facilitan el aprendizaje significativo de los estudiantes garantizando la calidad de la educación superior, esto fue también exteriorizado por los estudiantes quienes afirman que la aplicación y evaluación de técnicas por parte de los docentes es muy buena, en la entrevista a las autoridades coincide al decir que las técnicas ayudan a los estudiantes a resolver problemas matemáticos.

\section{Bibliografía}

Artigue, M., Douady, R., Moreno, L., \& Gómez, P. (1995). Ingeniería didáctica en educación Matemática. Grupo Editorial Iberoamericana, 97-140.

Ausubel, D. P. (1968). Educational psychology: A cognitive view. New York: Holt, Rinehart and Winston. trad. cast. Psicología educativa: un punto de vista cognoscitivo.

Beltrán, J. (1996). Procesos, estrategias y técnicas de aprendizaje. Revista Complutense de Educación.

Chevallard, Y., Bosch, M., \& Gascón, J. (1996). Estudiar Matemáticas: El eslabón perdido entre la enseñanza y el aprendizaje. Barcelona: ICEHORSORI. 
Coll, C., Martín, E., Mauri, T., Miras, M., Onrubia, J., Solé, I., \& Zabal, A. (1999). El contructivismo en el aula. Obtenido de (Microsoft Word SOL1311, Isabel y COLL, C\351sar. Los profesores y la concepci\363n.doc)

Edel Navarro, R. (2003). El rendimiento académico: concepto, investigación y desarrollo. Revista Electrónica Iberoamericana sobre Calidad, Eficacia y Cambio en Educación , 1-16.

Fernández De Haro, E. (s.f.). Estrategias de aprendizaje y técnicas de estudio. Obtenido de http://eoepsabi.educa.aragon.es/descargas/H_R ecursos/h_1_Psicol_Educacion/h_1.3.Apreder_ a_aprender/1.01.Estrategias_y_tecnicas.pdf

Gómez Gómez, M. (s.f.). Estrategias de aprendizaje y técnicas de estudio. Obtenido de https://www.edu.xunta.gal/centros/iesfelixmuri el/system/files/metodo+estudio.pdf

Julio, C. A. (2005). Las TICs y las Universidades: retos, posibilidades y preocupaciones. Revista de la Educación Superior Universidad de Sevilla.

Kolb, D.A. (1984). El aprendizaje experiencial: la experiencia como fuente de aprendizaje y desarrollo (Vol. 1). Englewood Cliffs, NJ: Prentice-Hall

Luis, T. E. (2013). Defendiendo lo colectivo: combatir el conformismo, promover la educcaión pública. Revista Interuniversitaria de Formación del Profesorado, 115-123.

Patricia, Z. M. (2008). Actas del II Congreso de Innovación Docente en Ciencias Jurídicas: Hacia el Espacio

Europeo de Educación Superior, Metodología de la enseñanza de la Historia del Derecho ante el Espacio Europeo de Educación Superior

Pozo, C. (1996). El fracaso académico en la universidad: Evaluación e intervención preventiva. Universidad Autónoma de Madrid

Sebastián, A., Ballesteros, B., \& Sánchez García, M. F. (s.f.). Técnicas de estudio. Obtenido de https://qinnova.uned.es/archivos_publicos/qwe b_paginas/3439/tecnicasdeestudio.pdf
Serrano Gónzález-Tejero, J. M., \& Pons Parra, R. M. (2011). El contructivismo hoy: enfoques contructivistas en educación. Revista Electrónica de Investigación Educativa, 1-28.

Tierno, B. (2012). Las mejores técnicas de estudio. Madrid: Grupo Planeta. 